\title{
POSSÍVEIS DESFECHOS RELACIONADOS AO USO DA ONDANSETRONA NA HIPERÊMESE GRAVÍDICA
}

\section{ARTIGO DE REVISÃO}

SILVA, Angel Adriany da ${ }^{1}$, TEIXEIRA, Gefferson Dias ${ }^{2}$, AZEVEDO, Marina Andrade de ${ }^{3}$, LUSO, Rafaela Pessoa Alves ${ }^{4}$, OLIVEIRA, Glauco Araujo de ${ }^{5}$, ZIETZ, Ana Carolina Gonçalves ${ }^{6}$, BRASIL, Vitória Borges ${ }^{7}$, VASCONCELOS, Isys Holanda Albuquerque de ${ }^{8}$, CARNEIRO, Caroline da Silva ${ }^{9}$, MAIA, Ryan Brendo Silva ${ }^{10}$

SILVA, Angel Adriany da. Et al. Possíveis desfechos relacionados ao uso da ondansetrona na hiperêmese gravídica. Revista Científica Multidisciplinar Núcleo do Conhecimento. Ano. 06, Ed. 10, Vol. 02, pp. 128-143. Outubro 2021. ISSN: 24480959, Link de acesso: https://www.nucleodoconhecimento.com.br/saude/hiperemesegravidica

${ }^{1}$ Discente do curso de Medicina da Pontifícia Universidade Católica do Paraná (PUCPR).

${ }^{2}$ Médico formada pela Universidade Federal do Ceará e Residente pela UFC na Santa Casa da Misericordia de Sobral.

${ }^{3}$ Discente do curso de Medicina do Centro Universitário INTA - UNINTA.

${ }^{4}$ Discente do curso de Medicina do Centro Universitário de Belo Horizonte - UniBH.

${ }^{5}$ Discente do curso de Medicina de... Universidade do Vale do Itajaí - UNIVALI.

${ }^{6}$ Discente do curso de Medicina de UFFS.

7 Discente do curso de Medicina de Universidade do Sul de Santa Catarina (UNISUL).

${ }^{8}$ Discente do curso de Medicina do Centro Universitário UNINTA, Sobral-Ce.

9 Discente do curso de Medicina da Universidade Estadual do Rio de Janeiro (UERJ).

${ }^{10}$ Discente do curso de Medicina da Unichristus.

RC: 98588

Disponível em: https://www.nucleodoconhecimento.com.br/saude/hiperemese-gravidica 


\section{RESUMO}

Introdução: Sintomas de náuseas e vômitos são comuns no primeiro trimestre da gestação, no entanto, algumas mulheres podem desenvolver hiperêmese gravídica, condição que traz esses sintomas de forma mais grave e recorrente, e muitas vezes refratária as medidas iniciais preconizadas atualmente. O cloridrato de Ondasetrona é um potente antiemético, porém até o momento não tem indicação franca em gestantes, e é prescrito apenas de forma off label e controversa. Objetivo: Analisar e discutir possíveis desfechos associados ao uso de ondansetrona durante a gravidez. Pergunta Problema: Tratando-se a gravidez de período delicado, é possível realizar a prescrição desse medicamento de forma segura nessas pacientes? Metodologia: Trata-se de uma revisão bibliográfica integrativa, retrospectiva do tipo qualitativa. Foi utilizado para a busca bibliográfica, o Portal da Biblioteca Virtual em Saúde (BVS), e o PubMed. Tal pesquisa foi realizada por meio de descritores disponíveis no DeCS (Descritores em Ciências da Saúde), sendo esses "Hyperemesis Gravidarum", "Hiperêmese Gravídica", "Pregnancy", "Grossesse" e "Ondansetron". Resultando em 13 artigos, avaliados pelo método de revisão integrativa, que contemplaram os objetivos do presente estudo. Resultados: No presente trabalho, foram incluídos 12 estudos associados às repercussões do uso da ondansetrona durante a gestação, entre eles $25 \%$ ( $n=3$ ) consideraram não haver evidências consistentes na literatura atual de que o uso da ondansetrona durante a gestação aumenta o risco do desfecho investigado. Por outro lado, $41 \%(\mathrm{n}=5)$ dos estudos incluídos defendem que há possibilidades para o uso, com ressalvas. Por fim, 3 dos estudos incluídos evidenciaram um potencial teratogênico no uso de ondansetrona durante a gestação. Conclusão: Os achados do estudo em relação ao uso de ondansentrona na gravidez constata que não há relação entre o fármaco e o aumento de risco de abortos espontâneos, também não há indícios sólidos em relação a efeito teratogênico e malformações congênitas. Porém é preciso atenção contínua durante a utilização, principalmente no decorrer do primeiro trimestre da gravidez. Ressalta-se ainda, a 
importância de estudos futuros que abordem acerca da relação da dose dessa substância e o real impacto da terapia no tratamento de hiperêmese gravídica.

Palavras-chave: Hiperêmese gravídica, Gestação, Cloridrato de Ondasetrona.

\section{INTRODUÇÃO}

No primeiro trimestre da gravidez, atingindo seu pico entre a 8 e 12 semana, é comum o aparecimento de sintomas como náuseas e vômitos. No entanto, quando essa condição se apresenta de forma mais grave e recorrente, ela recebe o nome de hiperêmese gravídica, que se caracteriza por vômitos persistentes que levam a uma perda de peso maior que $5 \%$ do peso pré-gravídico, sendo capaz de alterar o equilíbrio hidroeletrolítico e a nutrição da gestante. Tal condição ocorre em $0,3 \%$ a 3,0\% das gestações (BRASIL, 2012). Dessa forma, as gestantes fazem uso de medicamentos antieméticos para aliviar sintomas como os citados acima, sendo a Ondansetrona uma das drogas comumente prescrita, porém de forma off label, pois ainda não há recomendação que assegure que esse medicamento não trará prejuízos ao feto.

A substância cloridrato de Ondansetrona pertence à categoria dos antieméticos, sendo um medicamento usado na prevenção e tratamento de náuseas e vômitos associados à quimioterapia e radioterapia. E atualmente, seu uso tem sido considerado para tratar esses sintomas durante a gravidez. O medicamento em questão é um antagonista altamente seletivo dos receptores 5-HT3 da serotonina, no entanto seu mecanismo de ação ainda não é bem definido (ANDRADE, 2020).

Sabe-se apenas que esse receptor está presente em estruturas centrais, como na área postrema do bulbo, e receptores periféricos, especialmente no nervo vago. A área postrema é altamente relacionada ao centro do vômito, enquanto o nervo vago é responsável por inervação parassimpática de vísceras abdominais. Assim, a Ondansetrona pode estar relacionada à ação antagonista dessas estruturas centrais e periféricas, bloqueando esses receptores e, consequentemente, reduzindo a êmese (RANG et al., 2016).

RC: 98588

Disponível em: https://www.nucleodoconhecimento.com.br/saude/hiperemese-gravidica 
Porém, o conhecimento acerca do uso da Ondansetrona durante a gestação é relativamente dúbio, uma vez que até o momento, existe uma latente imprecisão na literatura médica mundial. Também não há evidências suficientes de que esse fármaco tem benefício superior em comparação com outros antieméticos, e têm suscitado dúvidas (SUAREZ et al., 2020). Logo, tratando-se a gravidez de período delicado, é possível realizar a prescrição desse medicamento de forma segura nessas pacientes?

Portanto, este artigo tem como objetivo esclarecer quais são os possíveis prós e contras associados ao uso de ondansetrona durante a gravidez, e se este estudo pode lançar as bases para a prescrição segura do medicamento neste caso.

\section{DESENVOLVIMENTO}

\subsection{METODOLOGIA}

Trata-se de uma revisão bibliográfica integrativa, retrospectiva do tipo qualitativa, destinada a analisar e descrever estudos nacionais e internacionais. Outrossim, é valido ressaltar que este estudo é adequado para aplicações práticas, visto que busca esclarecer quais os possíveis benefícios, malefícios ou riscos que estão associados ao uso da ondansetrona na gestação, com foco na hiperêmese gravídica.

Foram utilizadas como bases de dados, para a busca bibliográfica, o Portal da Biblioteca Virtual em Saúde (BVS), o PubMed e seus sites aliados. Primeiramente, foi realizada uma confirmação dos descritores apropriados na busca de artigos relevantes para o trabalho por meio do vocabulário disponível no DeCS (Descritores em Ciências da Saúde), sendo esses "Hyperemesis Gravidarum", "Hiperêmese Gravídica", "Pregnancy", "Grossesse" e "Ondansetron". Para o levantamento dos artigos, tais descritores possibilitaram, associado ao inglês e ao espanhol, a configuração da seguinte fórmula de busca: "(Hyperemesis Gravidarum) OR (Hiperêmese Gravídica) OR (Pregnancy) OR (Grossesse) AND (Ondansetron)".

$\mathrm{RC}: 98588$

Disponível em: https://www.nucleodoconhecimento.com.br/saude/hiperemese-gravidica 
Dessa forma, baseado no modelo de busca acima, foram encontrados um total de 97 artigos nos referentes bases de dados consultadas. Os critérios de inclusão utilizados foram: artigos publicados em português, inglês e espanhol; artigos disponíveis na integra que descrevessem tópicos relacionados a revisões abrangentes e artigos publicados e indexados nos referidos bancos de dados há pelo menos 5 anos, no intuito de enfocar as referências mais inéditas e relevantes, por conseguinte, apenas 19 dos 97 artigos foram incluídos. Além disso, foram excluídos os artigos indisponíveis na íntegra, repetidos e que não atendiam os objetivos da pesquisa, restando apenas 10 artigos que contemplavam os objetivos do estudo.

Diante disso, vale ressaltar que o pedido de pesquisa não é prático, portanto, há isenção ao Comitê de Ética em Pesquisa (CEP).

Nessa perspectiva, tanto a análise quanto a síntese dos dados extraídos dos artigos foram retratados de forma descritiva, visto que permitiu a configuração de uma tabela contendo as principais informações dos artigos que foram revisados na pesquisa, sendo essas: título, autores, ano de publicação, e os principais resultados, com fito de reunir o conhecimento gerado sobre o tema explorado na revisão.

\subsection{RESULTADOS}

No presente trabalho, foram incluídos 12 estudos associados às repercussões do uso da ondansetrona durante a gestação. Dentre eles, encontram-se 4 revisões sistemáticas (KAPLAN et al., 2019; PICOT et al., 2019; CARSTAIRS, 2016; DANIELSSON et al., 2018), sendo duas com metanálise (KAPLAN et al., 2019; PICOT et al., 2019). Ainda, 5 trabalhos trata-se de estudos de coorte retrospectivos (SUAREZ et al., 2020; LEMON et al., 2019; FEJZO et al., 2016; HUYBRECHTS et al., 2018; SUAREZ et al., 2020). Foram também analisadas duas revisões bibliográficas (ANDRADE, 2020; DANIELSSON et al., 2018). A Tabela 1 contém informações resumidas sobre cada um dos estudos.

\section{Características dos Estudos}

$\mathrm{RC}: 98588$

Disponível em: https://www.nucleodoconhecimento.com.br/saude/hiperemese-gravidica 
Ambas as revisões sistemáticas com metanálise incluíram estudos de coorte e estudos de caso-controle. Dentre os pré-requisitos usados por Kaplan et al. (2019) para a seleção de seus 8 trabalhos, estavam a exposição à ondansetrona durante a gravidez e a inclusão de um grupo controle. Enquanto Picot et al. (2019), se restringiu à investigação de malformações fetais associadas à exposição ao fármaco nas primeiras 20 semanas de gestação e incluiu 12 trabalhos.

Um total de 8 artigos atenderam aos critérios da revisão sistemática de Shaun (2016), que selecionou pesquisas originais, escritas em inglês, com grupo controle e exposição à ondansetrona durante o primeiro trimestre, investigando defeitos estruturais congênitos como resultado. Já Lavecchia et al. (2018) selecionou 10 estudos epidemiológicos entre artigos originais escritos em inglês, que relataram resultados fetais de exposição pré-natal à ondansetrona em humanos.

Já Andrade (2020), optou por avaliar Kaplan et al. (2019) e 4 outros artigos publicados na mesma época ou depois, que não haviam sido incluídos na referida metanálise. Também na revisão bibliográfica de Danielsson et al. (2018) agrupou os resultados dos estudos com ondansetrona em ratos, assim como resume informações sobre farmacocinética em ratos e humanos, de modo a permitir a comparação entre tais achados às gestações humanas.

Para seus 2 estudos de coorte, Suarez et al. (2020), selecionou gestantes que receberam prescrição de ondansetrona ou antieméticos comparativos (metoclopramida ou prometazina) durante as primeiras 20 semanas de gravidez, usando dados de prontuários eletrônicos de um sistema de saúde nos EUA. Para avaliar desfechos maternos, 2.677 gestações foram elegíveis, das quais $66 \%$ fizeram uso do medicamento, enquanto para a avaliação do risco de aborto espontâneo, 2.620 gestações foram elegíveis, das quais $65 \%$ foram expostas à ondansetrona.

Em seu estudo de coorte, Lemon et al. (2019) selecionou 33.677 gestações no Womens Hospital em Pittsburgh, dentre as quais 3733 (11\%) foram expostas à ondansetrona no primeiro trimestre. Também investigando repercussões da RC: 98588

Disponível em: https://www.nucleodoconhecimento.com.br/saude/hiperemese-gravidica 
exposição à ondansetrona no primeiro trimestre, Huybrechts et al. (2018) conduziu seu estudo no Medicaid Analytic extract, e selecionou 1816414 gestações, dentre as quais 88467 (4.9\%) foram expostas ao medicamento.

Em outra análise, por Fejzo et al. (2016) pacientes elegíveis foram recrutadas através de divulgação no site da Fundação de Pesquisa e Educação em Hiperêmese. Assim, foram coletados dados sobre o desfecho em 1.070 gestações expostas à ondansetrona, comparados aos desfechos em dois grupos controle: 771 gestações em mulheres com histórico de hiperêmese gravídica sem exposição à ondansetrona e 1555 gestações sem histórico de hiperêmese gravídica, nem exposição ao medicamento.

Tabela 1: Descrições dos estudos envolvendo o uso de ondansetrona na gestação.

\begin{tabular}{|c|c|c|}
\hline Título & $\begin{array}{l}\text { Autores, ano de } \\
\text { publicação }\end{array}$ & Principais resultados \\
\hline $\begin{array}{l}\text { Use of ondansetron during } \\
\text { pregnancy and the risk of } \\
\text { major congenital } \\
\text { malformations: } \\
\text { systematic review and } \\
\text { meta-analysis }\end{array}$ & $\begin{array}{l}\text { (KAPLAN et al., } \\
2019)\end{array}$ & $\begin{array}{l}\text { A ondansetrona pode ser utilizada } \\
\text { durante a gravidez quando } \\
\text { clinicamente indicado, pois há } \\
\text { baixos riscos de possíveis } \\
\text { malformações fetais. Entretanto, é } \\
\text { necessária vigilância contínua } \\
\text { durante o uso. }\end{array}$ \\
\hline $\begin{array}{l}\text { Ondansetron use in early } \\
\text { pregnancy and the risk of } \\
\text { late pregnancy outcomes }\end{array}$ & $\begin{array}{l}\text { (SUAREZ et al., } \\
2020)\end{array}$ & $\begin{array}{l}\text { Não há relação entre a } \\
\text { ondansetrona e o aumento de } \\
\text { distúrbios } \\
\text { gestacionais e risco de parto } \\
\text { prematuro, mas há um aumento } \\
\text { potencial no risco de fetos } \\
\text { pequenos para a idade gestacional. }\end{array}$ \\
\hline
\end{tabular}

RC: 98588

Disponível em: https://www.nucleodoconhecimento.com.br/saude/hiperemese-gravidica 
Ondansetron use in early (SUAREZ et al., Não houve relação entre o uso de pregnancy and the risk of 2020.) ondansetrona e o aumento no risco miscarriage

Major Congenital (ANDRADE, de aborto espontâneo.

Malformation Risk After 2020)

First

Trimester

Gestational Exposure to

Oral or Intravenous

Ondansetron

Em mulheres que possuem desequilíbrio eletrolítico associado à êmese ou hiperêmese gravídica, o uso de ondansetrona pode ser preocupante, pois aumenta o intervalo QTC.

Risk of malformation after (PICOT et al., A exposição de ondansetrona ondansetron in 2019) pregnancy: An updated systematic review and meta-analysis durante o primeiro trimestre da gravidez aumenta o risco de defeito no septo cardíaco, fissuras orofaciais, hérnia diafragmática e hipoplasia do coração esquerdo.

Ondansetron Use in (CARSTAIRS, Pregnancy and Birth 2016) Defects O uso de ondansetrona promove baixo risco geral de doenças congênitas. Apesar de baixo risco, esse medicamento deve ser usado caso haja refratariedade ao tratamento com outros medicamentos semelhantes.

Ondansetron use in the (LEMON et al., $O$ uso de altas doses de first trimester of 2019) pregnancy and the risk of neonatal ventricular septal defect ondansetrona está relacionado a um aumento no risco de comunicação intraventricular neonatal. Deve-se ponderar o uso em casos de vômitos e náuseas graves durante a gravidez. 


\begin{tabular}{|c|c|c|}
\hline $\begin{array}{l}\text { Ondansetron in } \\
\text { pregnancy and risk of } \\
\text { adverse fetal outcomes in } \\
\text { the United States }\end{array}$ & $\begin{array}{l}\text { (FEJZO et al., } \\
2016)\end{array}$ & $\begin{array}{l}\text { O uso de ondasetrona durante a } \\
\text { gravidez não aumenta o risco } \\
\text { teratogênico. }\end{array}$ \\
\hline $\begin{array}{l}\text { Association of Maternal } \\
\text { First-Trimester } \\
\text { Ondansetron Use With } \\
\text { Cardiac Malformations } \\
\text { and Oral Clefts in } \\
\text { Offspring }\end{array}$ & $\begin{array}{l}\text { (HUYBRECHTS } \\
\text { et al., 2018) }\end{array}$ & $\begin{array}{l}\text { No primeiro trimestre de gravidez o } \\
\text { uso de ondasetrona está } \\
\text { relacionado a um aumento do risco } \\
\text { de fissuras orais, mas não há } \\
\text { aumento do risco de malformações } \\
\text { cardíacas ou congênitas em geral. }\end{array}$ \\
\hline $\begin{array}{l}\text { indansetron } \\
\text { regnancy and the Risk of } \\
\text { Songenital Malformations: } \\
\text { Systematic Review }\end{array}$ & $\begin{array}{l}\text { (LAVECCHIA et } \\
\text { al., 2018) }\end{array}$ & $\begin{array}{l}\text { Há escassez de evidências de } \\
\text { aumento no risco de malformações } \\
\text { congênitas ao ser utilizado o } \\
\text { ondasetrona durante a gravidez. }\end{array}$ \\
\hline $\begin{array}{l}\text { Ondansetron and } \\
\text { teratogenicity in rats: } \\
\text { Evidence for a mechanism } \\
\text { mediated via embryonic } \\
\text { hERG blockade }\end{array}$ & $\begin{array}{l}\text { (DANIELSSON et } \\
\text { al., 2018) }\end{array}$ & $\begin{array}{l}\text { Há a possibilidade de um potencial } \\
\text { teratogênico no uso de } \\
\text { ondasetrona durante a gravidez. } \\
\text { Esse risco aumenta se a paciente } \\
\text { apresentar fatores de risco } \\
\text { adicionais, como hipocalemia. }\end{array}$ \\
\hline
\end{tabular}

Fonte: Próprio autor, 2020.

\section{Análise dos Resultados}

Entre os 12 estudos incluídos nesta revisão bibliográfica, 25\% ( $n=3)$ consideraram não haver evidências consistentes na literatura atual de que o uso da ondansetrona durante a gestação aumenta o risco do desfecho investigado. O estudo de coorte de Suarez et al. (2020), que visava estudar a relação entre o uso de ondansetrona e o risco de abortos espontâneos, concluiu não haver aumento do risco entre as usuárias do fármaco, comparadas as usuárias de outros antieméticos durante a gestação. $O$

$\mathrm{RC}: 98588$

Disponível em: https://www.nucleodoconhecimento.com.br/saude/hiperemese-gravidica 
estudo de coorte Fejzo et al. (2016) forneceu resultados que refutam a associação da ondansetrona ao aumento do risco teratogênico. Assim como o primeiro estudo citado, também comparou gestantes que usaram ondansetrona a gestantes que usaram outros antieméticos, ressaltando haver um aumento do risco de malformações fetais associado a hiperêmese gravídica por si só. Inclusive descreveu menos interrupções de gestação por hiperêmese gravídica entre as gestantes que usaram ondansetrona. Da mesma forma, a revisão sistemática de Lavecchia et al. (2018) conclui que há escassez de evidências que associem o uso de ondansetrona a malformações fetais. Contudo, destaca a necessidade de estudos adicionais para confirmar que o fármaco representa um tratamento seguro.

Por outro lado, $41 \%(n=5)$ dos estudos incluídos defendem que há possibilidades para o uso, com ressalvas. A metanálise de Kaplan et al. (2019) concluiu que a ondansetrona pode ser utilizada durante a gravidez quando clinicamente indicado, pois há baixos riscos de possíveis malformações fetais. Entretanto, recomenda vigilância contínua durante o uso. Já a revisão sistemática de Carstairs (2016) constatou que apesar do uso de ondansetrona promover baixo risco geral de doenças congênitas, o fármaco deve ser reservado para os casos refratários ao tratamento com medicamentos semelhantes.

O estudo de coorte de Suarez et al. (2020) visava a averiguar a associação do uso de ondansetrona a desfechos adversos maternos. Foi constatado que não há relação entre a exposição ao fármaco e o aumento de distúrbios hipertensivos gestacionais ou risco de parto prematuro, mas há um aumento potencial no risco de fetos pequenos para a idade gestacional. Já o estudo de coorte de Lemon et al. (2019) relatou que o risco é dose-dependente e que o uso de altas doses está relacionado a um aumento no risco de comunicação interventricular neonatal. Salientou que se deve ponderar o uso em casos de vômitos e náuseas graves durante a gravidez. Por sua vez, a revisão literária de Andrade (2020) frisou que o uso de ondansetrona pode ser preocupante em mulheres com desequilíbrio eletrolítico associado à êmese ou hiperêmese gravídica, pois aumenta o intervalo QT corrigido.

$\mathrm{RC}: 98588$

Disponível em: https://www.nucleodoconhecimento.com.br/saude/hiperemese-gravidica 
Por fim, 3 dos estudos incluídos evidenciaram um potencial teratogênico no uso de ondansetrona durante a gestação. O estudo de coorte de Huybrechts et al. (2018) evidenciou que, apesar do uso de ondansetrona no primeiro trimestre não estar relacionado a um aumento de malformações cardíacas ou congênitas em geral, configura um pequeno aumento no risco de fissuras orais. Por outro lado, a metanálise de Picot et al. (2019), não só reafirmou o risco aumentado de fissuras orofaciais, como também constatou que a exposição de ondansetrona durante o primeiro trimestre aumenta o risco de defeito no septo cardíaco, de hérnias diafragmáticas e de hipoplasia do coração esquerdo.

Ainda, os dados apresentados na revisão literária de Danielsson et al. (2018) sugerem que a ondansetrona usada na gestação apresenta potencial efeito teratogênico em humanos. Nos estudos conduzidos em ratos, conclui-se que o padrão de malformações são defeitos cardiovasculares e esqueléticos, que o risco é dosedependente e que aumenta na gravidez humana se fatores de risco adicionais estiverem presentes, como a hipocalemia devido a vômitos extensos.

\subsection{DISCUSSÃO}

\section{Desfechos Maternos: Cautela}

Como evidenciado neste artigo, um estudo mostrou que a ondansetrona possui uso off label no tratamento de grávidas com náuseas e vômitos considerados graves. Ademais, é considerada uma opção adicional em caso de persistência dos sintomas após tentativa de tratamento com outros antieméticos. Antes de considerar o uso de ondansetrona em pacientes que estejam no primeiro trimestre gestacional, é necessário que haja primeiramente a combinação de dois outros antieméticos. Se ainda assim houver insucesso na terapêutica, autoriza-se a introdução deste fármaco, considerando risco e eficácia na escolha entre administração oral ou intravenosa caso não haja hipovolemia (ACOG, 2018).

RC: 98588

Disponível em: https://www.nucleodoconhecimento.com.br/saude/hiperemese-gravidica 
O uso de ondansetrona é autorizado, contanto que seja indicado clinicamente. Apesar de evidenciar baixos riscos teratogênicos, sugere-se a observação contínua da gestante durante o tratamento. Este antiemético faz parte da classe $B$ de teratogênese da FDA, que agrupa drogas sem riscos aos fetos de animais, mas sem estudos em humanos (ROCHA et al., 2013; VAZ et al., 2019). Como seu uso em gestantes é controverso e os resultados dos estudos são conflitantes, faz-se necessário informar às usuárias os dados disponíveis e o baixo risco de malformações fetais. Além disso, deve-se analisar individualmente o uso de ondansetrona e suas vias de administração em mulheres que estejam com até 10 semanas de gestação, elencando riscos e benefícios. A cautela é pertinente devido ao risco, mesmo que pequeno, de anomalias cardiovasculares (ACOG, 2018).

Outro estudo contido nesta revisão mostrou que o uso de ondansetrona por mulheres que apresentam distúrbios eletrolíticos devido à hiperêmese gravídica pode ser preocupante, pois ocasiona o aumento do intervalo QT corrigido. A hiperêmese gravídica $(\mathrm{HG})$ é a forma mais grave da síndrome de náuseas e vômitos na grávida (NVG) e o uso de ondansetrona nesse contexto é assegurado por diversas sociedades do mundo (ANDRADE, 2020). Na fase de desidratação da doença, a gestante pode apresentar, além da alteração de doses de sódio e cloro, hipocalemia, o que gerar o prolongamento do intervalo QT (VAZ et al., 2019).

Ademais, outros estudos referem que o próprio uso do antiemético pode causar este desfecho. O medicamento pode atuar bloqueando o canal HERG $\mathrm{K}_{+}$, o que resulta no aumento do intervalo QT (RAPP et al., 2015). Além deste resultado, foram observados bradicardia, arritmias ventriculares e Torsades de Pointes nos usuários do medicamento (CHANDRAKALA et al., 2008; FREEDMAN et al., 2014; LEE et al., 2017).

A ondansetrona também pode causar aumento do QTc em pacientes com fatores de risco de arritmias subjacentes, hipomagnesemia, insuficiência cardíaca, uso de outros medicamentos que induzam essa alteração e uso de doses intravenosas múltiplas.

RC: 98588

Disponível em: https://www.nucleodoconhecimento.com.br/saude/hiperemese-gravidica 
Propõem-se que os usuários desta medicação façam monitoração eletrocardiográfica durante o tratamento (FREEDMAN et al., 2014). Além disso, recomenda-se que doses intravenosas únicas do antiemético não ultrapassem 16 mg (FDA, 2017; ACOG, 2018). No entanto, outro estudo mostra que se deve considerar o risco de aumento de QT mesmo em doses IV reduzidas (FREEDMAN et al., 2014; SAMANTA et al., 2014).

Por fim, outros estudo analisados no presente artigo mostraram ausência de relação do uso de ondansetrona com alguns desfechos. Um deles abordou a ausência da relação do antiemético com abortos espontâneos e outro mostrou que não há relação entre o uso da ondansetrona e o aumento de distúrbios hipertensivos gestacionais e risco de parto prematuro, porém há aumento do risco de fetos pequenos para a idade gestacional.

Da mesma forma, outro trabalho mostrou que não há associação do uso do medicamento com o aumento no risco de aborto espontâneo, natimorto ou qualquer outra anomalia congênita importante. Contudo, também evidenciaram que não há associação com o aumento do risco de parto prematuro e de feto nascido pequeno para a idade gestacional ou com baixo peso (PARKER et al., 2018). Nesse contexto, é perceptível a necessidade de novos estudos para esclarecimentos acerca dos resultados apresentados.

\section{Efeito Teratogênico e Malformações Congênitas}

Como já apresentado neste estudo, não há evidências sólidas acerca da relação do uso de ondansetrona com malformações congênitas. Os resultados de experimentos em ratos usando o antiemético no primeiro trimestre gestacional sugerem que pode haver efeito teratogênico em humanos, o que, de fato, foi constatado em dois outros estudos - também descritos nesta revisão. Um deles mostrou que seu uso aumenta apenas o risco de fissuras orais. O outro, além das fissuras orofaciais, relatou o aumento do risco de defeito no septo cardíaco, de hérnia diafragmática e de hipoplasia do coração esquerdo. No entanto, outros trabalhos desta revisão refutaram o risco RC: 98588

Disponível em: https://www.nucleodoconhecimento.com.br/saude/hiperemese-gravidica 
teratogênico, relataram baixo risco geral de malformações fetais e afirmaram haver escassez de evidências sobre a relação do uso de ondansetrona com esse desfecho.

Com efeito, o fármaco atravessa a placenta e é possível detectá-lo no tecido do feto. Em Parker et al. (2018) e Zambelli-Weiner et al. (2019) evidenciaram em seus trabalhos que não há associação com um alto risco de malformações congênitas, mas pode existir um discreto aumento absoluto no risco de fenda palatina e malformações cardiovasculares, principalmente no septo. Apesar destes resultados, há necessidade de confirmação de eventos congênitos específicos, haja vista que os dados em humanos são conflitantes. Além disso, ainda se estuda o risco de grandes malformações congênitas neste contexto (ACOG, 2018).

Portanto, é evidente a necessidade de estudos adicionais para atestar a segurança deste medicamento. De qualquer forma, permanece a recomendação de prescrição de ondansetrona para gestantes, preferencialmente em casos refratários ao tratamento de náuseas e vômitos, sempre prestando esclarecimento sobre o baixo risco de malformações (VAZ et al., 2019).

O estudo de Lemon et al. (2019) indica que a dose é importante no desfecho de complicação fetal. Altas doses do antiemético podem resultar em aumento no risco de comunicação interventricular no feto. Como já exposto, há um discreto aumento absoluto no risco de malformações fetais, principalmente no septo cardíaco. Contudo, mostrou-se que a relação dose-dependente é evidente no aumento do intervalo QT das gestantes (ACOG, 2018). Desse modo, seria necessário um estudo específico para confirmação acerca da relação da dose de Ondansetrona com desfechos fetais.

\section{CONSIDERAÇÕES FINAIS}

Os achados do estudo em relação ao uso de ondansentrona na gravidez constata que não há relação entre o fármaco e o aumento de risco de abortos espontâneos, já que não foram encontradas evidências entre as usuárias do medicamento, comparadas as gestantes que utilizaram outros antieméticos. Porém é preciso atenção contínua

RC: 98588

Disponível em: https://www.nucleodoconhecimento.com.br/saude/hiperemese-gravidica 
durante a utilização, principalmente no decorrer do primeiro trimestre da gravidez. Em relação a efeito teratogênico e malformações congênitas, não há indícios sólidos a respeito do uso de ondansetrona com malformações congênitas. Ressalta-se ainda, a importância de estudos futuros que abordem acerca da relação da dose dessa substância com desfechos fetais e malformações fetais e o real impacto da terapia no tratamento de hiperêmese gravídica.

\section{REFERÊNCIAS}

ACOG. COMMITTEE ON PRACTICE BULLETINS-OBSTETRICS. ACOG Practice Bulletin No. 189: Nausea And Vomiting Of Pregnancy. Obstetrics \& Gynecology, [s. l.], v. 131, ed. 1, p. e15-e30, 2018. Disponível em: https://journals.Iww.com/greenjournal/Abstract/2018/01000/ACOG_Practice_Bulletin_ No_189_Nausea_And.39.aspx. Acesso em: 21 jan. 2021.

ANDRADE, Chittaranjan. Major Congenital Malformation Risk After First Trimester Gestational Exposure to Oral or Intravenous Ondansetron. The Journal of Clinical Psychiatry, [s. I.], v. 81, ed. 3, 2020. DOI 10.4088/JCP.20f13472. Disponível em: https://www.psychiatrist.com/jcp/psychopharmacology/major-congenitalmalformation-associated-with-gestational-exposure-to-ondansetron-weighing-therisks/. Acesso em: 28 dez. 2020.

CARSTAIRS, Shaun D. Ondansetron use in pregnancy and birth defects: a systematic review. Obstetrics \& Gynecology, [s. I.], v. 127, ed. 5, p. 878-883, 2016. Disponível em:

https://journals.Iww.com/greenjournal/Abstract/2016/05000/Ondansetron_Use_in_Pr egnancy_and_Birth_Defects_A.10.aspx. Acesso em: 15 jan. 2021.

CHANDRAKALA, $R$ et al. Ondansetron induced fatal ventricular tachycardia. Indian journal of pharmacology, https://www.ncbi.nlm.nih.gov/pmc/articles/PMC2792616/, v. 40, ed. 4, 2008. DOI https://dx.doi.org/10.4103\%2F0253-7613.43168. Disponível 
em: https://www.ncbi.nlm.nih.gov/pmc/articles/PMC2792616/. Acesso em: 28 dez. 2020.

DANIELSSON, B et al. Ondansetron and teratogenicity in rats: Evidence for a mechanism mediated via embryonic hERG blockade. Reproductive Toxicology, [ $s$. I.], v. $81, \quad$ p. 237-245, 2018. Disponível em: https://www.sciencedirect.com/science/article/pii/S0890623818300972. Acesso em: 15 jan. 2021.

FDA (United States government). Center for Drug Evaluation and Research. FDA Drug Safety Communication: New information regarding QT prolongation with ondansetron (Zofran). Food and Drug Administration (FDA), [s. I.], 2017. Disponível em: https://www.fda.gov/drugs/drug-safety-and-availability/fda-drug-safetycommunication-new-information-regarding-qt-prolongation-ondansetron-zofran. Acesso em: 3 dez. 2020.

FEBRASGO (Brasil). Nota Febrasgo: Ondansetrona no tratamento das náuseas e vômitos na gravidez. Febrasgo, [s. I.], 4 out. 2019. Disponível em: https://www.febrasgo.org.br/pt/noticias/item/873-ondansetrona-no-tratamento-dasnauseas-e-vomitos-na-gravidez. Acesso em: 8 jan. 2021.

FEJZO, Marlena S. et al. Ondansetron in pregnancy and risk of adverse fetal outcomes in the United States. Reproductive Toxicology, [s. I.], v. 62, p. 87-91, 2016. Disponível em: https://www.sciencedirect.com/science/article/pii/S0890623816300910. Acesso em: 15 jan. 2021.

FREEDMAN, Stephen B. et al. Ondansetron and the risk of cardiac arrhythmias: a systematic review and postmarketing analysis. Annals of Emergency Medicine, [s. I.], v. 64 , ed. 1 , p. 19-25.e6, 2014. Disponível em: 10.1016/j.annemergmed.2013.10.026. Acesso em: 28 dez. 2020. 
HUYBRECHTS, K. F. et al. Association of Maternal First-Trimester Ondansetron Use With Cardiac Malformations and Oral Clefts in Offspring. JAMA, v. 320, n. 23, p. 2429, dez. 2018. Disponível em: https://jamanetwork.com/journals/jama/articleabstract/2718793. Acesso em: 15 jan. 2021.

KAPLAN, Y. C. et al. Use of ondansetron during pregnancy and the risk of major congenital malformations: A systematic review and meta-analysis. Reproductive Toxicology, v. 86, p. 1-13, jun. 2019. Disponível em: https://www.sciencedirect.com/science/article/pii/S089062381830529X. Acesso em: 15 jan. 2021.

LAVECCHIA, M. et al. Ondansetron in Pregnancy and the Risk of Congenital Malformations: A Systematic Review. Journal of Obstetrics and Gynecology Canada, v. 40, n. 7, p. 910-918, jul. 2018. Disponível em: https://www.sciencedirect.com/science/article/pii/S1701216317307570.Acesso em: 15 jan. 2021.

LEE, Danny et al. Torsades de Pointes after Ondansetron Infusion in 2 Patients. Texas Heart Institute Journal, [s. I.], v. 44, p. 366-369, 2017. DOI 10.14503/THIJ-16-6040. Disponível em: 10.14503/THIJ-16-6040. Acesso em: 28 dez. 2020.

LEMON, L. S. et al. Ondansetron use in the first trimester of pregnancy and the risk of neonatal ventricular septal defect. International Journal of Epidemiology, v. 49, n. 2, p. 648-656, dez. 2019. Disponível em: https://academic.oup.com/ije/articleabstract/49/2/648/5682456. Acesso em: 15 jan. 2021.

MINISTÉRIO DA SAÚDE (Brasil). Departamento de Ações Programáticas Estratégicas. Gestação de alto risco: manual técnico. 5․ ed. Brasilia: Editora do Ministério da Saúde, 2012. 302 p. ISBN 978-85-334-1767-0. Disponível em: https://bvsms.saude.gov.br/bvs/publicacoes/manual_tecnico_gestacao_alto_risco.pdf . Acesso em: 28 dez. 2020.

RC: 98588

Disponível em: https://www.nucleodoconhecimento.com.br/saude/hiperemese-gravidica 
PARKER, Samantha E et al. Ondansetron for treatment of nausea and vomiting of pregnancy and the risk of specific birth defects. Obstetrics \& Gynecology, [s. I.], v. 132, ed. 2, p. 385-394, 2018. DOI 10.1097/AOG.0000000000002679. Disponível em: https://journals.Iww.com/greenjournal/Fulltext/2018/08000/Ondansetron_for_Treatme nt_of_Nausea_and_Vomiting.16.aspx. Acesso em: 28 dez. 2020.

PARKER, Samantha $E$ et al. Ondansetron for treatment of nausea and vomiting of pregnancy and the risk of specific birth defects. Obstetrics \& Gynecology, [s. I.], v. 132, ed. 2, p. 385-394, 2018. DOI 10.1097/AOG.0000000000002679. Disponível em: https://journals.Iww.com/greenjournal/Fulltext/2018/08000/Ondansetron_for_Treatme nt_of_Nausea_and_Vomiting.16.aspx. Acesso em: 28 dez. 2020.

PICOT, C. et al. Risk of malformation after ondansetron in pregnancy: An updated systematic review and meta-analysis. Birth Defects Research, v. 112, n. 13, p. 9961013, 2020. Disponível em: https://onlinelibrary.wiley.com/doi/abs/10.1002/bdr2.1705. Acesso em: 15 jan. 2021.

RANG, H P et al. Rang \& Dale: Farmacologia. 8․ ed. [S. I.]: Elsevier Editora Ltda, 2016. 1939 p. ISBN 978-85-352-6500-2. Disponível em: https://cssjd.org.br/imagens/editor/files/2019/Abril/Farmacologia.pdf. Acesso em: 28 dez. 2020

RAPP, Jonathan $\mathrm{H}$ et al. Bradycardia after intravenous ondansetron with asystole on rechallenge: a case report. Hospital pharmacy, [s. I.], v. 50, ed. 10, p. 918-921, 2015. DOI https://doi.org/10.1310/hpj5010-918. Disponível em: https://journals.sagepub.com/doi/10.1310/hpj5010-918. Acesso em: 28 dez. 2020.

ROCHA, Rebeca Silveira et al. Consumo de medicamentos, álcool e fumo na gestação e avaliação dos riscos teratogênicos. Rev. Gaúcha Enferm.,, Porto Alegre, v. 34, ed. 2, p. 7-45, 2013. Disponível em: https://seer.ufrgs.br/rgenf. Acesso em: 28 dez. 2020.

RC: 98588

Disponível em: https://www.nucleodoconhecimento.com.br/saude/hiperemese-gravidica 
SAMANTA, Sukhen et al. Intraoperative pulseless ventricular tachycardia after Ondansetron. Journal of Anaesthesiology Clinical Pharmacology, [s. I.], v. 30, ed. 2, p. 293-294, 2014. DOI https://dx.doi.org/10.4103\%2F0970-9185.130123. Disponível em: https://www.ncbi.nlm.nih.gov/pmc/articles/PMC4009666/. Acesso em: 28 dez. 2020.

SUAREZ, Elizabeth A. et al. Ondansetron use in early pregnancy and the risk of miscarriage. Biblioteca Wiley Online, [s. I.], 2020. Disponível em: https://onlinelibrary.wiley.com/doi/10.1002/pds.5151. Acesso em: 3 dez. 2020.

SUAREZ, Elizabeth A. et al. Ondansetron use in early pregnancy and the risk of late pregnancy outcomes. Biblioteca Wiley Online, [s. I.], 2020. Disponível em: https://onlinelibrary.wiley.com/doi/10.1002/pds.5151. Acesso em: 3 dez. 2020.

VAZ, Jorge Oliveira et al. Hiperêmese Gravídica. In: TRATADO de Obstetrícia Febrasgo. [S. I.: s. n.], 2019. p. 768-785.

ZAMBELLI-WEINER, April et al. First trimester ondansetron exposure and risk of structural birth defects. Reproductive Toxicology, [s. I.], v. 83, p. 14-20, 2019. DOI 10.1016/j.reprotox.2018.10.010. em: https://www.sciencedirect.com/science/article/pii/S0890623818301230. Acesso em: 3 dez. 2020.

Enviado: Setembro, 2021

Aprovado: Outubro, 2021. 